\title{
Interview avec Elisabeth Ansen Zeder, membre de l'ASP
}

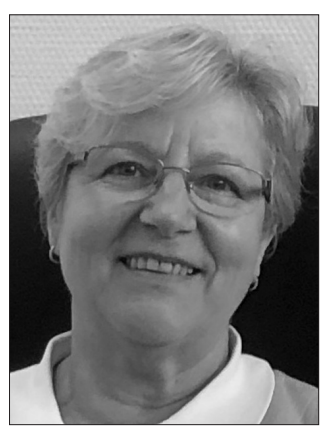

Elisabeth Ansen Zeder

\section{Quelles ont été vos motivations pour}

choisir la profession de psychothérapeute?

Des motivations profondément humanistes. J'ai grandi à Strasbourg, dans un climat proche de la terre mais où quelques difficultés familiales ont été le moteur d'une recherche de compréhension de la vie psychique. C'est donc mon expérience de vie qui a été ma principale motivation.

\section{Quel est votre contexte/parcours} professionnel?

Éducatrice de la petite enfance à l'origine, je viens du terrain d'accompagnement direct. J'ai travaillé comme éducatrice spécialisée, dans différents établissements et notamment à l'aide à l'enfance en France. J'ai aussi travaillé plusieurs années dans léducation nationale en France et en Belgique, avant d'arriver en Suisse en 1999. Durant la période où je résidais en Rhône-Alpes jai effectué un DEA en psychologie et psychopathologie clinique à Lyon, sous la direction des professeurs René Roussillon et Jacques Hochmann, en 1991. J'ai accompli une psychanalyse lacanienne, me suis formée en analyse transactionnelle et, plus tard, j’ai effectué un Doctorat en psychologie clinique sous la direction de la professeure Evelyne Thommen. Dans ce cadre, ma recherche portait sur les processus de résilience des frères et sœurs de personnes présentant une déficience intellectuelle. J'ai aussi accompli un cursus en analyse existentielle et en logothérapie. Compte tenu de son intérêt pour la clinique et de sa reconnaissance par l'OMS, je me suis aussi formée à l'EMDR. Mon parcours professionnel s'est ainsi déployé dans différents contextes.

\section{Travaillez-vous en tant que psychothéra-} peute indépendant(e) en cabinet privé et/ ou (éventuellement en outre) en tant que psychothérapeute délégué(e) ?

Je travaille comme psychothérapeute libérale, de façon indépendante et en délégation d'une médecin psychiatre. Les deux me semblent intéressants et complémentaires, en fonction des situations. En effet, certains patients ont besoin d'une médication et le lien direct avec un médecin psychiatre prescripteur est donc facilitant.

Exercez-vous une autre profession, une autre occupation en plus de la psychothérapie? Ayant effectué un doctorat en psychologie clinique, j’enseigne la psychologie du développe- ment et de l'apprentissage dans les contextes de formation d'enseignants. Auparavant auprès de l'Institut de pédagogie curative et maintenant à la Haute école pédagogique de Fribourg depuis 2003.

\section{Quelle est votre spécialisation?}

J'aime la diversité des approches et me suis formée dans différentes directions dans l'optique d'un humanisme intégré. Mais j'ai beaucoup élaboré les questions du "Spiritual Care " anglo-saxon et me suis formée en logothérapie. La recherche de sens est fondamentale dans mon approche. Mon travail est orienté sur les ressources des patients, avec un fondement psychanalytique qui trouve sa perspective dans une visée psychosomatique et noétique.

\section{Vous sentez-vous satisfait(e) de votre situation professionnelle?}

Très contente, oui, même si j’ai parfois un peu trop de travail vu mes différents mandats. Cela me donne toutefois souplesse et ouverture, ravive élan et fraîcheur, même si jarrive bientôt au bout de ma carrière.

\section{Souhaiteriez-vous que quelque chose soit différent?}

J'aimerais que la reconnaissance de notre métier soit meilleure. La réforme en vue va dans le bon sens, mais je crains d'un côté le risque de surcharge administrative par la masse de rapports qu'il s'agira de produire, et de l'autre que le tarif soit lissé vers le bas. Je souhaiterais que la prestation de psychothérapie soit pleinement reconnue et rétribuée pour l'importance qu'elle a.

\section{Avez-vous un souhait à adresser à votre association, l'ASP?}

Je souhaite que l'ASP prenne plus de poids, et puisse notamment constituer un véritable interlocuteur influent aux côtés de la FSP qui est très présente en Romandie. J'apprécie le traitement humain, le sens du service que je ressens à l'ASP et il m'importe que notre faîtière continue d'exister, et même se développe, compte tenu de la place spécifique qu'elle occupe. Son ouverture à la diversité des cursus en fait une association d'une grande richesse, où sont réunies de nombreuses personnes aux parcours diversifiés, venant d'horizons différents. 


\section{Vous sentez-vous représenté(e)}

et reconnu(e) dans votre association

professionnelle, l'ASP ?

Oui, si ce n'est le poids trop fort ressenti dans d'autres faîtières, en particulier dans ma région. Je souhaiterais que l'ASP ait plus de poids en Romandie et accorde plus d'importance aux régions de Suisse non germanophones. Les séances se tiennent souvent à Zurich, ou plus loin, mais il est presque impossible, dès lors, pour un Romand, de s'y rendre facilement. Des localisations plus centrales constitueraient un signal d'intérêt plus explicite.

\section{Quel serait votre priorité si vous faisiez} partie du comité de l'ASP?

De donner force et poids à l'ASP, et de faire les efforts de location intégrative évoqué pour que tout membre, quel que soit son lieu de rattachement, se sente pleinement membre de l'ASP.

\section{Y a-t-il une fonction dans l'ASP \\ que vous occuperiez volontiers?}

Compte tenu de la proche fin de mon parcours professionnel et de mes multiples engagements, non.

Quelle serait selon vous la situation idéale pour les psychothérapeutes

dans l'environnement politique donné?

Une véritable reconnaissance libérale avec une possibilité d'exercer en pouvant compter sur une reconnaissance salariale à la mesure du long cursus de formation du psychothérapeute.

\section{Quelle vision avez-vous}

pour votre quotidien professionnel?

Lobjectif est de savoir pleinement être dans l'ici et maintenant de la relation, dans le respect de l'autre, mais aussi de soi. Et prochainement, je me réjouis d'intervenir dans le colloque interna-

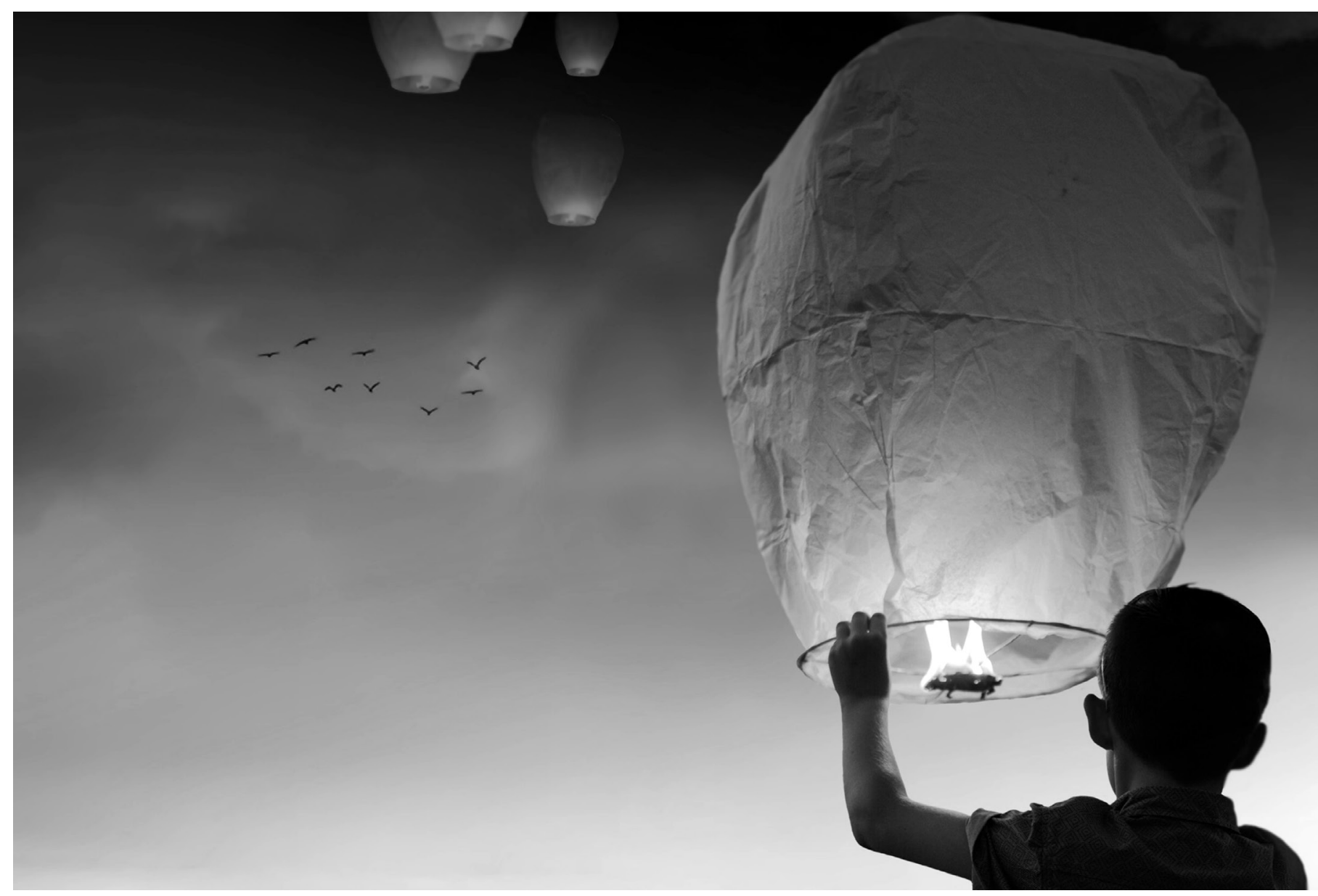


tional la «clinique du sens " qui se tiendra les 14/15 novembre prochain sur le campus de Lausanne https://www.unil.ch/issr/clinique-du-sens

Elisabeth Ansen Zeder, Dr. en psychologie, est Psychologue FSP/Psychothérapeute ASP, Psychothérapeute et formatrice-chercheuse,
Membre de l'ASP depuis 2013 et elle a une cabinet de psychothérapie. Sites : www.relationaide.ch | https://www. hepfr.ch/users/elisabethzederfreducanet2ch

L'entrevue a été menée par Sandra Feroleto et enregistrée par écrit.

74 Demande | à jour! Évolution de la profession de psychothérapeute 10 